\title{
Cortical Blindness as an Unusual Adverse Drug Reaction
}

\author{
Caroline Wilson a, , Darren Leaning ${ }^{\mathrm{a}}$, Kate Shankland ${ }^{\mathrm{a}}$, Tobias Langheinrich ${ }^{\mathrm{a}}$, \\ Ruth Batty ${ }^{\text {a }}$ Penella J. Woll ${ }^{\text {a }}$
}

\begin{abstract}
Cortical blindness is a frightening and unusual adverse drug reaction that can be difficult to diagnose. It is characteristic of reversible posterior leukoencephalopathy syndrome (RPLS), which has been described as an idiosyncratic reaction to a variety of anti-cancer drugs. Here we describe a case of RPLS in a patient receiving treatment with an immunoconjugate, and review its association with anti-cancer drugs.
\end{abstract}

Keywords: Adverse drug reaction; Anton syndrome; IMGN901; Immunoconjugate; Leukoencephalopathy; Merkel cell cancer

\section{Case Report}

A 62-year-old woman was diagnosed with multifocal CD56positive Merkel cell carcinoma (MCC) of the right lower leg in August 2008. She had well controlled insulin dependent type II diabetes mellitus, rheumatoid arthritis, hypertension, ischemic heart disease, peripheral vascular disease and pre eclampsia in two of three pregnancies. There were no distant metastases, and she was treated with six cycles of chemotherapy with carboplatin and etoposide, achieving a partial response. Within six weeks of stopping chemotherapy, however, the tumors had regrown and new lesions had appeared. The patient therefore entered a Phase I dose escalation study of IMGN901 (Immunogen), an immunoconjugate of the cy-

Manuscript accepted for publication August 25, 2010

a Departments of Clinical Oncology, Neurology and Radiology, Sheffield Teaching Hospitals, NHS Foundation Trust, Sheffield, UK ${ }^{\mathrm{b}}$ Corresponding author: Weston Park Hospital, Sheffield S10 2SJ, UK. Email: caroline.wilson@sth.nhs.uk

doi:10.4021/jmc47w totoxic maytansiniod DM1 and a humanised murine monoclonal antibody huN-901, which selectively delivers the cytotoxic to CD56+ cells [1]. She received IMGN901 $60 \mathrm{mg} /$ $\mathrm{m}^{2}$ by intravenous infusion on days $1-3$ of a planned three week cycle.

On day 15 she was admitted to hospital with lower back pain and constipation. On admission, she was apyrexial with a blood pressure of $130 / 70 \mathrm{mmHg}$, pulse rate $80 / \mathrm{min}$, oxygen saturations $98 \%$ on air and a respiratory rate of $18 / \mathrm{min}$. The only abnormality on blood analysis, which included full blood count, urea and electrolytes, glucose, amylase, liver function, vasculitic screen and thyroid function, was a mildly raised creatinine of $110 \mu \mathrm{mol} / \mathrm{L}$. Twenty four hours later, she suddenly experienced nausea and profuse vomiting associated with headache. She became acutely confused and dis-

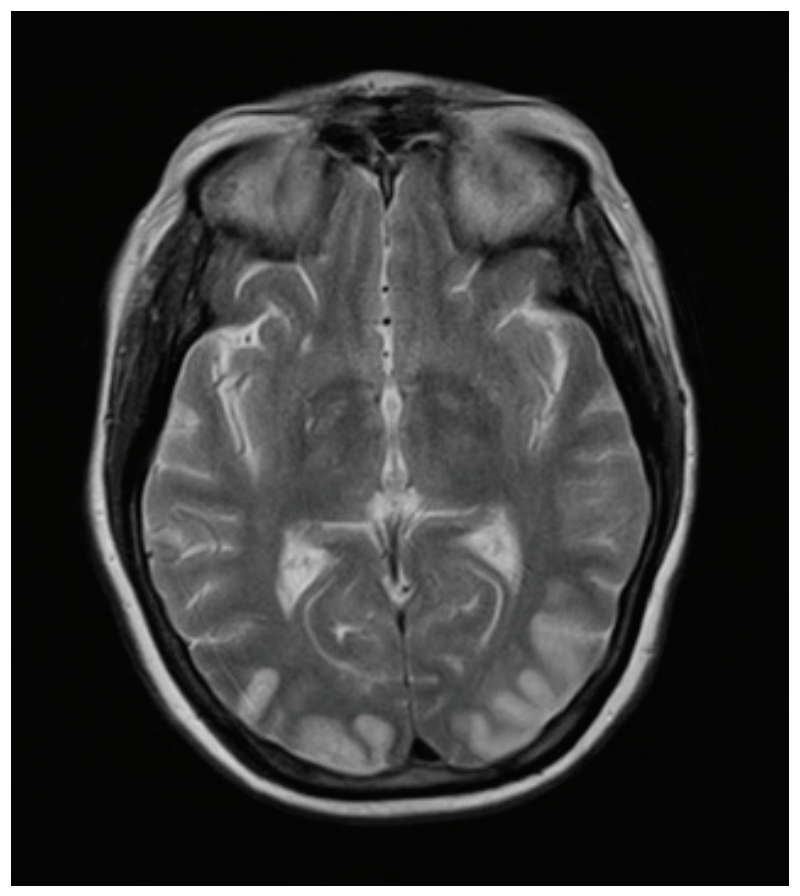

Figure 1. MR brain revealed bilateral symmetrical areas of increased T2 weighted signal involving the cortex and subcortical white matter of the parietal and occipital lobes. 


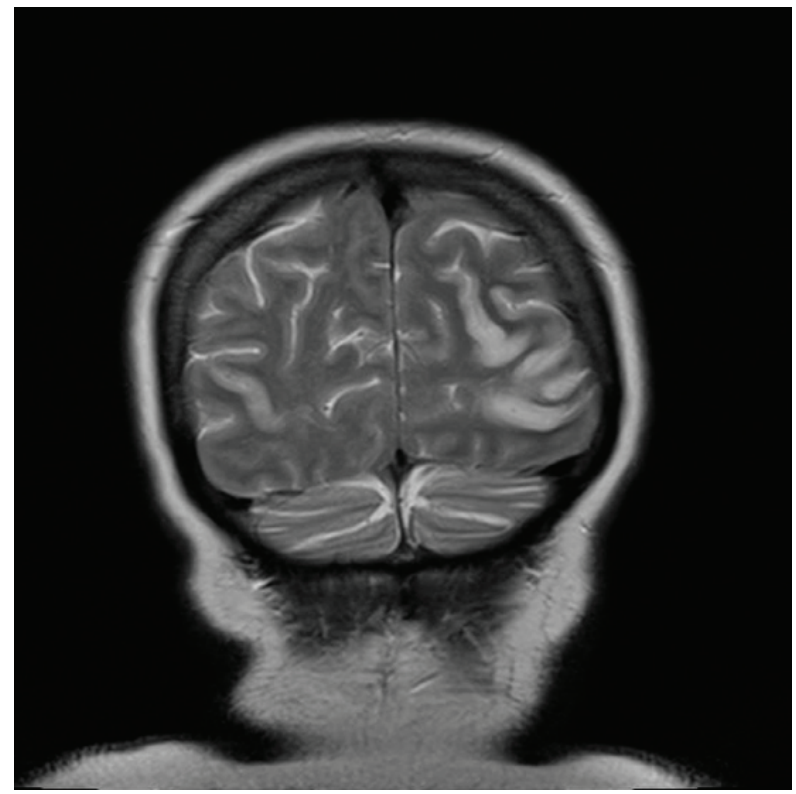

Figure 2. MR brain revealed bilateral symmetrical areas of increased T2 weighted signal involving the cortex and subcortical white matter of the parietal and occipital lobes.

orientated, unable to recognise her husband. She was hemodynamically stable. A CT head scan was non-diagnostic due. Lumbar puncture revealed normal pressure, clear CSF, negative for malignant cells, red cell count of $3 \times 10^{6} / \mathrm{L}$, white cell count of $9 \times 10^{6} / \mathrm{L}$. The CSF protein was mildly raised at $659 \mathrm{mg} / \mathrm{L}$. The CSF glucose was $3.3 \mathrm{mmol} / 1$ compared to blood glucose $5.6 \mathrm{mmol} / \mathrm{l}$. Gram stain was negative, culture negative, PCR for varicella, herpes and entero viruses was

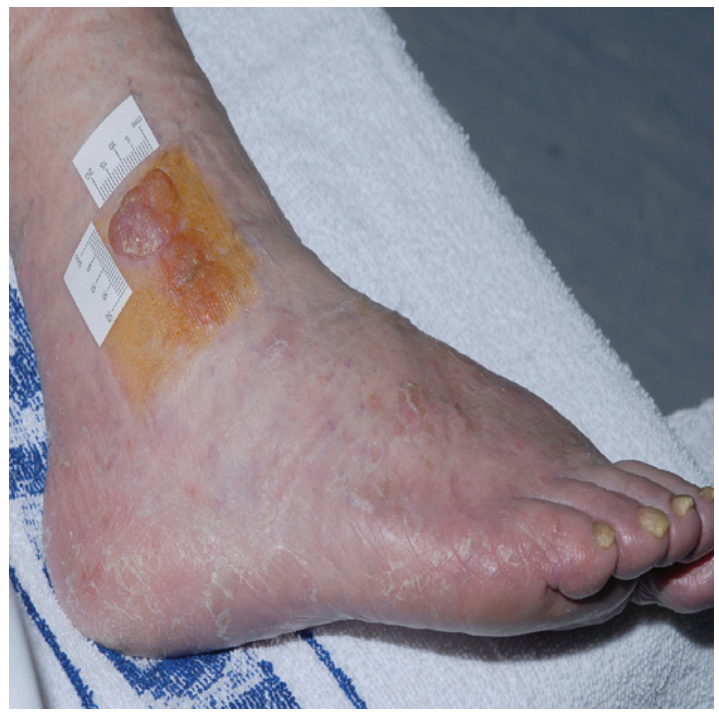

Figure 3. Right ankle prior to IMGN901 treatment. negative. Broad spectrum intra venous antibiotics and antivirals were instituted with a working diagnosis of encephalitis. Within 48 hours of admission, day 17, the patient had become more lucid and a full neurological examination was possible. This revealed cortical blindness but the patient was unaware of this and denied any visual loss. There was a full range of eye movements and no relative afferent pupillary defect, implying a functional optic nerve. The remainder of the cranial nerve examination was normal and fundoscopy unremarkable. Peripheral muscle tone, power and reflexes were normal. Her gait was hesitant due to visual impairment but she had no cerebellar signs. An MR brain was performed which revealed bilateral symmetrical areas of increased T2 weighted signal involving the cortex and subcortical white matter of the parietal and occipital lobes, the changes demonstrated a restrictive diffusion pattern implying that the MR findings were acute, and not for example chronic secondary to previous pre-ecplampsia (Fig. 1 and 2). The appearances were typical of reversible posterior leukoencephalopathy syndrome (RPLS).

The patient improved slowly on supportive measures and was discharged home 16 days after admission. Her vision had partially recovered, but she could not read news paper print. Her distance vision was blurred causing unsteadiness and necessitating the use of a walking frame. Over the next six months, her vision continued to slowly improve. She resumed writing, was able to draw up her insulin and walk independently with a stick. She was reviewed by an ophthalmologist three months after discharge and was found to have visual acuity of 6/12 in the right eye and 6/9 in the left eye. Visual field testing revealed a large infero-nasal field defect with normal fundi and no diabetic retinopathy. The field defect was attributed to cortical association area dam-

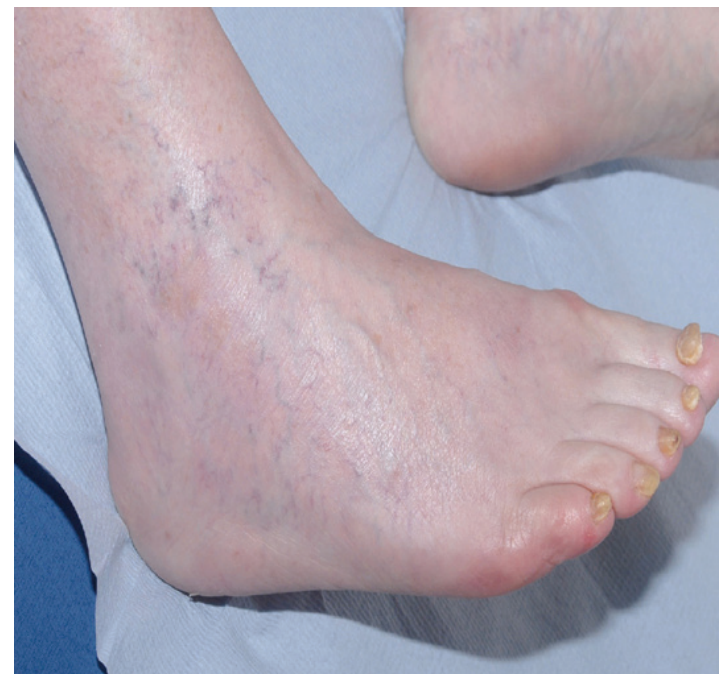

Figure 4. Right ankle 5 months after the single cycle of IMGN901treatment. 
age secondary to infarction which can occur in association with RPLS. She declined further imaging of her head. No further IMGN901 was given and she has had no further anticancer treatment. Remarkably, the MCC tumors completely resolved. Figures 3 and 4 show the right ankle prior to IMGN901 treatment (Fig. 3) and 5 months after the single cycle of IMGN901 treatment (Fig. 4). She remained in clinical complete remission 12 months later.

\section{Discussion}

Reversible posterior leukoencephalopathy syndrome (RPLS) is a rare neurological condition initially described by Hinchey et al in 1996. It has been described in sporadic case reports, but its incidence is unknown. The diagnosis is based on typical clinical and radiological findings. The clinical presentation frequently includes headache, altered mental state, visual disturbance including cortical blindness, and seizures [2]. The onset is usually subacute, with most patients experiencing a change in alertness as an early symptom. Temporary confusion and agitation may alternate with lethargy which may lead to stupor or coma. Abnormalities of visual perception are common and can range from blurred vision to cortical blindness. It is important to note that Anton's syndrome can develop, in which the patient denies their blindness, and it may be days before relatives, or health care staff realize that the patient cannot see [3]. Clinicians should be alert to this in suspected cases, and undertake a thorough neurological examination. RPLS is usually reversible within 2 weeks on removal of the causative agent.

Characteristic radiological abnormalities on CT or MR

Table 1. Anti-cancer Agents Reported to be Associated With RPLS

\begin{tabular}{ll} 
Cytotoxics & Biological Response Modifiers \\
\hline Cisplatin & Bevacizumab \\
Cyclosporin & Dexamethasone \\
Cyclophosphamide & Immunoglobulin \\
Cytarabine & Interferon $\alpha$ \\
Gemcitabine & Rituximab \\
Methotrexate & Sorafenib \\
Oxaliplatin & Sunitinib \\
& Tacrolimus \\
\hline
\end{tabular}

brain scans include edema involving the white matter in the posterior portions of the cerebral hemispheres, particularly in the parieto-occipital regions [2, 4]. Involvement of other areas of the brain has also been reported, including the frontal lobes, grey matter or brain stem. Treatment involves removal of the causative agent, and tight control of blood pressure. Although RPLS is often reversible, lesions can progress to infarcts causing permanent damage. Diffusion weighted images can differentiate between edema and cellular damage, and thus predict cases where damage may be irreversible

The etiology of RPLS is controversial, and thought to be associated with a potential effect on the vascular endothelium that can lead to vasogenic edema. It can also be associated with uncontrolled hypertension, pre-eclampsia, collagen vascular disorders, renal failure, thrombotic microangiopathy, hypercoagulopathy and a variety of anti-cancer agents (Table 1), all of which can alter the blood brain barrier and thus create a more susceptible CNS microvasculature [2, 5]. The direct cause of damage to the vascular endothelium is not known, but possibilities include a toxic effect or via vasoconstriction caused by elevated endothelin. It is not associated with any specific malignancy and no single anti-cancer agent has been found to be consistently associated with RPLS [5]. It appears to be an idiosyncratic adverse effect with no dose relationship. No previous cases of RPLS with IMGN901 have occurred, with over 150 patients receiving the drug to date. Toxicities include fatigue, peripheral sensory neuropathy and headache/meningitis-like symptoms. The incidence of headaches has been reduced by increasing the infusion time and pre-medication with steroids, and the meningitis-like symptoms have not been observed with the use of these prophylactic measures.

A number of theories have been proposed for the pathogenesis of RPLS associated with specific antineoplastic agents. Bevacizumab, a potent inhibitor of VEGF disrupts angiogenesis and can induce vasospasm, hypertension and proteinuria: sunitinib, an inhibitor of VEGFR-2 and PDGFR- $\beta$, causes capillary endothelial damage and can induce hypertension. Intravenous immunoglobulin can also cause vasogenic edema, cerebral vasospasm and serum hyperviscosity. Soussain et al [5] comment that although most antineoplastic agents do not cross the blood brain barrier, in vivo-experiments have demonstrated toxic effects of cisplatin and cytarabine on CNS progenitor cells, which persist after discontinuation of treatment. The combination of antineoplastic agents may further alter blood brain barrier permeability making combination regimens potentially more toxic to the CNS. Other proposed methods of CNS injury include; cytokine induced damage, free radical production, accumulation of DNA damage, reduced telomerase activity, inhibition of DNA repair enzymes and immunological dysfunction. In this case, either the antibody or the cytotoxic could be responsible for RPLS. A direct effect is unlikely be- 
cause RPLS developed two weeks after the final drug dose, when serum levels would be undetectable.

In conclusion, increased awareness of RPLS amongst oncologists is imperative for early diagnosis and treatment. No single antineoplastic agent has been consistently linked to RPLS, and there is no clear dose response relationship. The early withdrawal of the causative agent is necessary to prevent long term neurological sequelae. Because of its propensity for the posterior brain, particularly the occipital lobes, RPLS can cause cortical blindness. This can prove difficult to diagnose, particularly when clinical symptoms are denied by the patient (Anton's Syndrome). There is currently no prophlylactic or curative treatment for RPLS and the pathophysiology remains poorly understood. Further work is required to elucidate the causes, risk factors, prevention and treatment of this potentially catastrophic complication of commonly used chemotherapeutic agents.

\section{References}

1. Woll P, Fossella F, O'Brien M et al. Phase I Study of IMGN901 (BB10901) in patients with CD56 -positive solid tumors. European Journal Cancer Suppl 2008; 6: 162.

2. Lee VH, Wijdicks EF, Manno EM, Rabinstein AA. Clinical spectrum of reversible posterior leukoencephalopathy syndrome. Arch Neurol 2008;65(2):205-210.

3. Prigatano GP. Anosognosia: clinical and ethical considerations. Curr Opin Neurol 2009.

4. Sanders TG, Clayman DA, Sanchez-Ramos L, Vines FS, Russo L. Brain in eclampsia: MR imaging with clinical correlation. Radiology 1991;180(2):475-478.

5. Soussain C, Ricard D, Fike JR, Mazeron JJ, Psimaras D, Delattre JY. CNS complications of radiotherapy and chemotherapy. Lancet 2009;374(9701):1639-1651. 